\title{
Effectiveness and safety of monoclonal antibodies for metastatic colorectal cancer treatment: systematic review and meta-analysis
}

\author{
Bruno Rosa, Jose Paulo de Jesus, Eduardo L de Mello, Daniel Cesar and Mauro M Correia \\ Instituto Nacional de Câncer, Rio de Janeiro 20230-130, Brazil
}

Correspondence to: Daniel Cesar. Email: danielcesar@live.com

\begin{abstract}
Background: The effectiveness of chemotherapy (CT) for select cases of metastatic colorectal cancer (MCRC) has been well established in the literature, however, it provides limited benefits and in many cases constitutes a treatment with high toxicity. The use of specific molecular biological treatments with monoclonal antibodies (MA) has been shown to be relevant, particularly for its potential for increasing the response rate of the host to the tumour, as these have molecular targets present in the cancerous cells and their microenvironment thereby blocking their development. The combination of MA and CT can bring a significant increase in the rate of resectability of metastases, the progression-free survival (PFS), and the global survival (GS) in MCRC patients.

Objective: To assess the effectiveness and safety of MA in the treatment of MCRC.

Methods: A systematic review was carried out with a meta-analysis of randomised clinical trials comparing the use of cetuximab, bevacizumab, and panitumumab in the treatment of MCRC.

Results: Sixteen randomised clinical trials were selected. The quality of the evidence on the question was considered moderate and data from eight randomised clinical trials were included in this meta-analysis. The GS and PFS were greater in the groups which received the MA associated with CT, however, the differences were not statistically significant between the groups (mean of 17.7 months versus 17.1 months; mean difference of 1.09 (Cl: $0.10-2.07) ; p=0.84$; and 7.4 versus 6.9 months. mean difference of 0.76 (Cl: $0.08-1.44) ; p=0.14$ respectively). The meta-analysis was not done for any of the secondary outcomes.
\end{abstract}

Conclusion: The addition of MA to CT for patients with metastatic colorectal cancer does not prolong GS and PFS.

Keywords: monoclonal antibody, cetuximab, bevacizumab, panitumumab, colorectal neoplasia, metastatic colorectal cancer

Published: $15 / 10 / 2015$

Received: 11/04/2015

ecancer 2015, 9:582 DOI: 10.3332/ecancer.2015.582

Copyright: @ the authors; licensee ecancermedicalscience. This is an Open Access article distributed under the terms of the Creative Commons Attribution License (http://creativecommons.org/licenses/by/3.0), which permits unrestricted use, distribution, and reproduction in any medium, provided the original work is properly cited. 


\section{Introduction}

Colorectal cancer ( $\mathrm{CRC}$ ) is the third most common type of malignant neoplasia diagnosed in men and women in the world, and is also the second principal cause of death from cancer in the United States, following only lung cancer [1-7]. In Brazil, according to data from the National Cancer Institute (INCA), the estimates for the year 2014 indicate the occurrence of 15,070 new cases of CRC in men and 17,530 in women. These figures correspond to an estimated risk of 15.44 new cases in each 100,000 men and 17.24 in each 100,000 women, constituting the fourth most common absolute type of cancer in the Brazilian population [8].

CRC develops slowly, over several years of progression through different molecular and cytological stages, becoming a carcinoma with the potential for invasion and metastasis [4, 9]. Metastasis to the liver is the second most common (the first is to the lymph nodes) and affects approximately half of the patients with CRC, where only $11.7 \%$ survive for about five years following the diagnosis [7, 10, 11]. Surgical resection continues to be the only procedure which provides a possible cure [12], however, less than $20 \%$ of patients have liver metastases of colorectal tumours (LMCRC) which are potentially resectable [13].

For several decades, the basis for systemic treatment of advanced LMCRC has been chemotherapy (CT), composed of 5-fluorouracil (5-FU) in combination with leucovorin (LV), for which the therapeutic result has been the achievement of a global survival (GS) of 12 months [7, 10]. More recent agents, such as irinotecan (IR) and oxaliplatin (OX) improve the response and survival rates [7, 10, 14, 15]. It is well established in the literature that the administration of pre-operative CT to patients with initially non-resectable LMCRC can reduce the size of the tumours such that curative resection becomes possible (conversion chemotherapy), and can also reduce the risk of recurrence following resection. In addition, it helps to predict the prognosis by determining the tumour response, which in the end helps to exclude non-responders and select the best adjuvant therapy [5, 12]. Randomised clinical trials and meta-analyses have shown improvement in the response and survival rates with first-line chemotherapy (5-Fu/LV) in patients with LMCRC $[7,12,16]$, such as the addition of oxaliplatin to this protocol (FOLFOX) significantly improves the response rate and progression-free survival (PFS) in advanced cases of LMCRC [7].

Meanwhile, a phase III study of the European Organisation for Research and Treatment of Cancer (EORTC) concludes that pre-operative CT (FOLFOX) can cause damage to the remaining liver. According to this study, regimens including OX are associated with an increased risk of vascular lesions (such as severe hepatic sinusoidal obstruction, also called 'blue liver'), while regimens including IR have been associated with an increased risk of steatosis and steatohepatitis. Secondary analyses also show that the administration of six cycles of FOLFOX was associated with a moderate increase in the risk of reversible complications following surgery [12, 17].

Tol et al showed that the combination of capecitabin + IR (CAPIRI) was associated with a greater incidence of grade 3-4 toxicity, in comparison with the combination of 5-FU/LV + IR (FOLFIRI) [10]. In another study, pre-operative CT based on FOLFOX/FOLFIRI for LMCRC were not significantly associated with an increase in GS or PFD [18]. Nevertheless, the introduction of new therapeutic strategies made possible an increase in the expected GS for patients with non-resectable LMCRC to 22 months [19].

Although several mechanisms determine the action of conventional CT, they all seek to act on the cells, blocking the replication of their DNA. As they are not specific for the cancer cells, these mechanisms are often associated with toxicity for normal tissues [13, 20]. As a result, the specific biological molecular therapies for LMCRC have achieved great relevance in the last few years in the search for a more selective action [21]. The introduction of a new class or agents called monoclonal antibodies (MA) have provided additional benefits $[10,13,20]$. These agents bind to the binder (protein which binds to a receptor) or the extracellular domain of the receptor itself. That is this group of agents inhibits the means for the transduction of signals by means of the tyrosine kinase receptors which are necessary for the growth of the cancer cells (these have molecular targets present in their structure and microenvironment), thus blocking their development $[10,13,20]$. The identification of these agents is changing the treatment of LMCRC. Two types of MAs were approved by the US Food and Drug Administration (FDA) and by the European Medicines Agency (EMA) for clinical purposes in LMCRC: the endothelial vascular growth factor inhibitors (anti-VEGF), represented by bevacizumab; and the epidermal growth factor receptor inhibitors (anti-EGFR), represented by cetuximab and panitumumab [10,14].

Cetuximab (Erbitux ${ }^{\circledR}$ ) is a monoclonal chimeric G1 immunoglobulin (lgG1) which recognises and binds to the extracellular domain of the EGFR, blocking the activation of this receptor [13, 20, 22-24]. It was approved after an improvement in the survival and inverse 
chemoresistance was demonstrated when administered with IR in phase II trials [23], also presenting evidence of their efficacy and safety when added to FOLFIRI, as a first-line intervention for carriers of CCRm who have wild-type KRAS [24]. Approximately $20 \%$ of all colorectal cancers contain wild KRAS (the absence of a mutation on the KRAS oncogene) [25, 26, 27,28] and only tumours which express the wild-type KRAS can be candidates for cetuximab, while the patients with mutant KRAS are considered resistant. It can be used as a monotherapy or in combination with primary, secondary, or tertiary configurations in patients with LMCRC [22].

The results of an analysis of the mutational state of KRAS done by the FIRE-3 study confirmed prior results showing that first-line CT (FOLFIRI) associated with cetuximab achieved a good response in terms of the global response rate and GS in the majority of CCRm carriers with wild KRAS (exon 2), extended to those with wild KRAS exons 3/4, and also wild NRAS oncogenes exons 2/3/4. Meanwhile, a subgroup of patients with mutated KRAS did not obtain a similar benefit, showing an improvement in PFD only following treatment with FOLFIRI associated with another MA. The results for PFD showed a similar tendency, patients with wild-type RAS and BRAF mutation obtained a discrete advantage with FOLFIRI associated with cetuximab, when compared with FOLFIRI alone, and when compared with FOLFIRI associated with another monoclonal antibody (FIRE III).

A recent meta-analysis which compared cetuximab + irinotecan versus chemotherapy alone, when used as a first-line treatment for LHCRC, demonstrated that the addition of cetuximab was statistically superior to isolated chemotherapy in terms of the general response to treatment, the GS time and the PFS [24]. Another study demonstrated that cetuximab can also preserve the quality of life (QOL) of patients with advanced CRC [29]. Among the most commonly observed adverse events in these studies were cutaneous toxicity (rash) $[13,24]$, reactions to the infusion, hypomagnesemia, fatigue, abdominal pain, nausea, and diarrhoea [13]. Among the inoperable LHCRC, the combinations of cetuximab + FOLFOX-6 (oxaliplatin/5-FU/LV) and cetuximab + FOLFIRI significantly increased the resectability of the metastases, including $\mathrm{R} 0$ resections [24].

Panitumumab (Vectibix ${ }^{\circledast}$ ), a G2 kappa immunoglobulin and a human recombinant antibody, was established as an effective monotherapy in patients with LMCRC resistant to fluoropyrimidine, OX, and IR [14]. The results of two studies show that panitumumab improves the results when added to FOLFOX or FOLFIRI among patients with CCRm and wild-type KRAS [14]. Meanwhile, their application to mutated KRAS and NRAS genes (the latter a homologue of the viral RAS gene of the neuroblastoma) generates an inverse response, as these genes are capable of predicting the absence of the benefit from the addition of panitumumab to CT, (Douillard 2013). When administered as a first- or second-line treatment in combination with CT, panitumumab is shown to be significantly better than chemotherapy alone in terms of PFS in patients with metastatic colorectal cancer (MCRC) [30].

It has an acceptable tolerability profile when administered as a monotherapy or in combination with CT. Its toxicity is related to alterations in the skin and seems to present a low risk of immunogenicity [30]. Panitumumab received advance approval from the Food and Drug Administration (FDA) based on the improvement in PFS and an independent response rate of $8 \%$, similar to that observed with other active agents in the advanced phase of the illness [31].

In a similar manner to panitumumab, clinical trials indicate that bevacizumab (Avastin ${ }^{\circledR}$ ), a humanised recombinant antibody, in combination with 5-FU + folic acid and bevacizumab in combination with IR/5-FU/LV are clinically effective, when compared with standard first-line CT for the treatment of LMCRC [32]. These results seem to extend to second-line CT. A recent multicentric phase III study assessed the continued use of bevacizumab associated with second-line CT in patients with LMCRC who had progress after use of the drug with firstline CT [33]. The GS was statistically greater in the intervention group when compared with the group that received CT only [33]. Finally, Masi et al concluded that bevacizumab can be used safely with FOLFOXIRI (IR, OX, 5-FU, and folinate) after assessing the efficacy and safety of this plan in 57 patients with non-resectable LHCRC. The treatment achieved promising results in terms of PFS (equal to $74 \%$ at ten months $(95 \% \mathrm{Cl} 62-85)$ [34].

Although there is evidence of the efficacy of this new class of drugs, the best pre-operative regimen is still not well established. Optimisation of results and reduction of toxicity are goals to be achieved [24]. The primary objective of this study is to assess the effectiveness and safety of MAs in the treatment of metastatic colorectal cancer, in addition to their effects. 


\section{Methods}

This study is a systematic review of the literature with meta-analysis. The following inclusion criteria were used: randomised clinical trials (RCTs) of adult participants ( $>18$ years of age) of both sexes with a confirmed histological diagnosis of CRC and evidence of metastatic illness, assessing MAs associated with any CT (intervention group-at least one of the three drugs-cetuximab, bevacizumab, or panitumumab) versus CT without MAs (control group) or placebo, applied to metastatic illness.

We consider oral, systemic, or intravenous intervention associated with CT or anti-angiogenic agents (or even a combination of these treatment modalities) for the treatment of MCRC.

The criteria for exclusion were: other primary study types (quasi-randomised, cohort, and case-control clinical trials, among others); RCTs with participants < 18 years of age; studies with volunteers; studies with a single branch of treatment; studies of non-pharmacological interventions (eg. surgery, radiation therapy).

The primary outcomes were GS and PFS, both measured in months. GS is understood as the time elapsed from the date of initial diagnosis of the metastasis and the date of death because of any cause [35]. PFS is the period of time from the randomisation to any progression of the illness or death, whichever happens first [15, 36]. We used the Response Evaluation Criteria in Solid Tumours (RECIST) version 1.1 [37] to define progression in this study, which was characterised as an increase of at least $20 \%$ in the major diameter of the target lesion, taking the lowest measurement of the major diameter, verified at the start of treatment or the appearance of one or more new lesions as a reference. The secondary objectives were the toxicity of the treatment, according to the Common Toxicity Criteria or the National Cancer Institute $(\mathrm{NCl})$ of the United States, including the rate of deaths related to treatment. Other secondary objectives were: the time for progression, defined as the time from the randomisation and the objective tumour progression (not including deaths), which was also assessed according to the RECIST criteria; along with the rate of resectability, and the conversion rate.

The assessment of QOL represents an assessment of the impact of a health condition and its treatment on all of the pertinent and important aspects of the life of the patient, such as for example physical, social, psychological, symptoms, and perceptions.

\section{Search for identification of studies}

We identified all of the relevant studies (Figure 1), independently of year or language. RCTs were identified through a systematic search in the following electronic databases [Appendix 1]: Cochrane Colorectal Cancer Group, The Cochrane Central Register of Controlled Trials (CENTRAL); MEDLINE and EMBASE. The search for unpublished RCTs or those in progress was from their metaRegister of Controlled Trials [38] using the following search terms: monoclonal antibodies; mAb; anti-VEGF; anti-EGRF; bevacizumab OR, avastin OR. panitumumab OR. vectibix cetuximab OR. erbitux OR. colorectal neoplasms OR. colorectal cancer OR. colorectal carcinomas. The search was performed by two authors (Rosa BR, and Correia MM), independently and the concordance between them was assessed using the Kappa correlation coefficient. The last search was performed in March of 2014. The search strategy in PUBMED was the following: ('Colorectal neoplasms/drug therapy' [MESH] OR 'Colorectal neoplasms/therapy [MESH]) AND (cetuximab [Title/Abstract] OR panitumumab [Title/Abstract]) OR, bevaczumab OR (CHEMOTHERAPY [Title/Abstract]).

\section{Analysis and synthesis}

\section{Assessment of the risk of bias in the studies included}

The risk of bias in the studies included was assessed independently by means of a domain-based assessment, considering the following domains associated with the risk of bias: generation of the allocation sequence (method used to generate the allocation sequence); blinding of the allocation (method used for blinding the allocation sequence, in order to know if the allocations of interventions could be predicted in advance); blinding of participants, researchers, and assessors of results (means used to prevent the participants in the study from becoming aware of the intervention that they or any other participant may have received); and selective recording of results (a situation in which part of the study results may have been published). Each of these items was classified into categories: 'Yes', 'No', or 'Uncertain', indicating low, high, or unknown risk of bias, respectively. We contacted the authors of the studies when we found unclear or missing data. The results of these analyses are given in a chart of the risk of bias (Figure 2). 


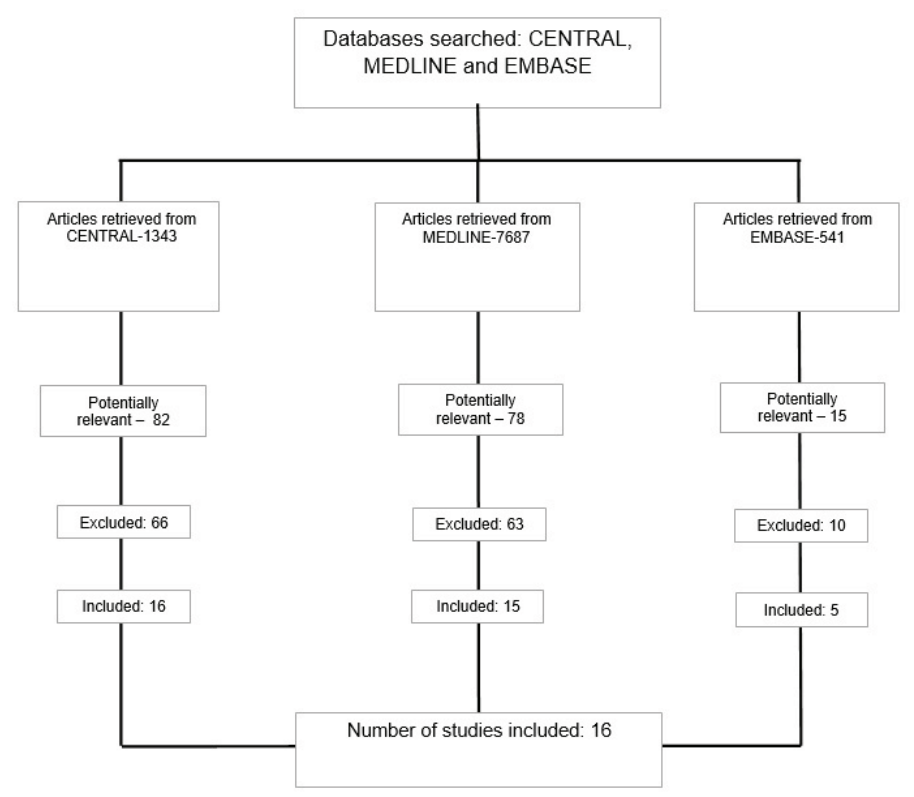

Figure 1. Study selection flowchart.

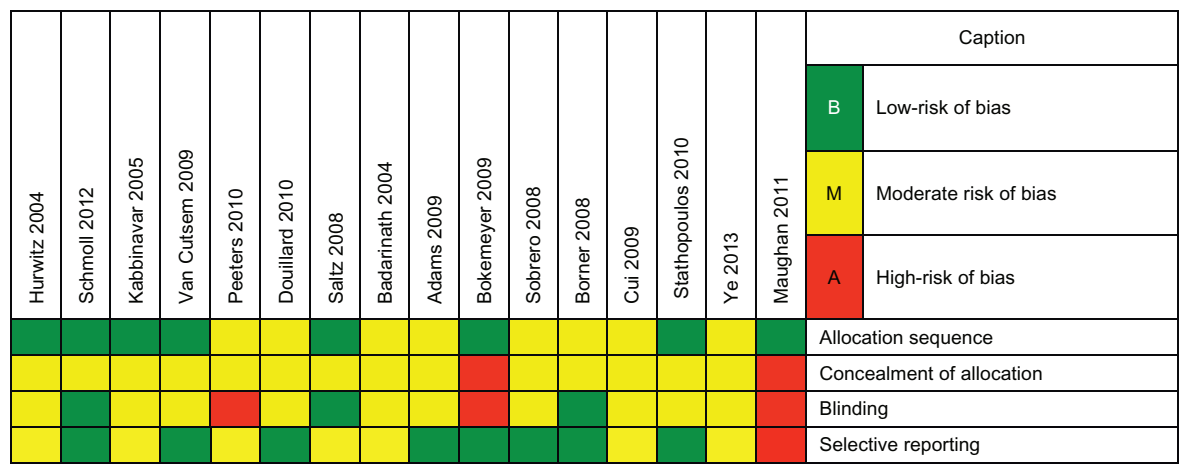

Figure 2. Summary of the risk of bias in the included studies.

Measures of the effect or treatment, synthesis of data, and statistical heterogeneity among the studies

We compared the opposing data using the relative risk (RR) and the consistent data using the means difference. All of the estimates had a confidence interval of $95 \%(\mathrm{Cl})$, where $p<0.05$ was statistically significant. All of the analyses were done using the Review Manager 5 (RevMan 5) software, from the Cochrane Team. Meta-analysis was done when at least one of the same outcomes was assessed in at least two RCTs. Analysis of the subgroup was done based on the KRAS mutation state (group 1: KRAS mutated; group 2: wild KRAS). Studies which included the two types of KRAS were not included in this type of analysis.

\section{Results}

\section{Description of the studies and flow chart}

The flow chart for the selection of the studies is shown in Figure 1. The reviewers finally agreed to include sixteen [16] published RCTs on the MAs in question, involving 12,015 participants and with a Kappa concordance coefficient of 0.5 (satisfactory) [39-54]. 


\section{Characteristics of the selected studies}

These RCTs assessed cetxiumab (eight RCTs-5517 participants), bevacizumab (six RCTs-4129 participants) and panitumumab (two RCTs-2369 participants) associated with CT. Data from eight RCTs was included in the meta-analyses, with a total of 7128 participants. The median age of the participants was 61.2 years.

Cetuximab was assessed in eight studies (5517 participants) [44-49, 53, 54]. Of these, five added cetuximab to first-line CT. In two of these five, cetuximab was associated with OX + fluoropyrimidine versus the same strategy without MA, with a total of 2434 participants [44, 46]. While both had performed the studies with three branches of treatment, the analyses were considered in two groups. Another two studies (375 participants) assessed the role of cetuximab when associated with FOLFOX-4 (OX + leucovorin + 5-FU in a bolus) [47, 43]. The last added MA to FOLFIRI (irinotecan + leucovorin + 5-FU in an infusion), randomising the groups with or without cetuximab, with a total of 1198 participants [45]. Finally, one RCT assessed whether FOLFOX-6 associated with cetuximab would be more effective than FOLFIRI in 138 participants randomised into two groups [54]. In another study with 1298 participants for whom prior treatment with first-line CT had failed, (FOLFOX - OX + leucovorin + 5-FU (in an infusion)) or irinotecan (IR) was associated or not with cetuximab [48]. Another study used cetuximab + XELOX (OX + capecitabin) versus XELOX in 74 participants [49].

Bevacizumab was assessed in six RCTs (4129 participants) [39-41, 50-52]. In three of these (1097 participants), the combination of IR, 5-FU, and leucovorin (LV) were used with or without bevacizumab [39, 50, 51]. Two other studies used bevacizumab associated with different CT schemes [40, 41]. The first RCT (209 participants) compared bevacizumab to a placebo, both associated with the 5-FU + LV scheme [40]. The other study (1401 participants) [41] compared bevacizumab to a placebo, both associated with XELOX and FOLFOX-4 (bevacizumab + XELOX or FOLFOX-4 versus placebo + XELOX or FOLFOX-4). This study was initially performed with two treatment branches. Finally, the RCT which included 1422 participants compared bevacizumab to cediranib (an inhibitor of the tyrosine kinase of the vascular endothelial growth factor receptor), both combined with first-line CT [52]. Then panitumumab was assessed in two other studies totaling 2369 participants. In a study with 1186 participants this was associated or not with FOLFIRI [42].

\section{Risk of bias}

Overall, the methodological quality of the studies was considered to be moderate. The allocation concealment method was not mentioned in any of 16 RCTs; it was the item with the highest risk of bias. Patients were not blinded in three of the eight RCTs that were included in the meta-analyses, totaling 3153 participants [42, 44, 47]. Randomisation was properly performed in eight RCTs; its status is unknown in the remaining eight studies. For this domain, the risk of bias was considered to be moderate. The practice of selective reporting was clearly identified in only one publication [44]. However, the $\mathrm{Cl}$ of some means were not published in some RCTs, which prevented these studies from being included in the meta-analyses [39-42, 50, 52, 54].

Figure 3 presents the RCTs distribution in a funnel plot for OS (Figure 3A) and PFS (Figure 3B) outcomes. The funnel plot can indicate the presence of publication bias. A funnel plot is a scatter plot of the effect estimates of individual studies in some measure of size or precision of each study. In the absence of heterogeneity between studies, a possible dispersion will be because of sampling variation between clinical trials, and the graph may resemble a symmetrical inverted funnel. A triangle centered on the fixed effect estimation will extend for 1.96 standard error on each side and will include $95 \%$ of the studies if no bias is present under the assumption of fixed effect (which is the true treatment effect in each study). Otherwise, there may be asymmetry, which will indicate some sort of heterogeneity (statistical, methodological, or clinic) and/or publication bias.

\section{Excluded studies}

After all the initial exclusions, 65 studies were classified as potentially relevant [55-119]. However, in a more rigorous evaluation, these studies did not match the inclusion criteria of the present review and were discarded. Nineteen were excluded because they had only one treatment group [55-73], 31 had at least one MA in more than one treatment group [74-104], three did not have CT associated with 
the intervention [105-107], three were retrospective studies [108-110], two were not RCTs [111, 112], three were secondary data analysis [113-115], two were locally severe disease [116, 117], one did not include metastatic disease [118], and the final study assessed the intervention after metastatic surgery [119].

\section{Effect of interventions}

Figures 4 and 5 present the effect of interventions regarding OS (Figure 4) and PFS (Figure 5).

\section{Secondary outcomes}

\section{Toxicity and safety}

Treatment toxicity (including the death rate), the proportion of patients eligible for resection, the time to progression, and the conversion rate were assessed in seven RCTs [39, 44, 46, 47, 52-54]. None of the above criteria could be assessed by meta-analysis, because the authors did not publish the Cls of the percentages and/or means for each of the groups. Treatment-related deaths were evaluated only in two RCTs (both with cetuximab), totaling 27 deaths among 2434 participants (1.1\%), and 14 in control groups (CT only) versus 13 deaths in the intervention groups (CT + cetuximab) [44, 46]. Only one RCT reported no death by any cause [54].

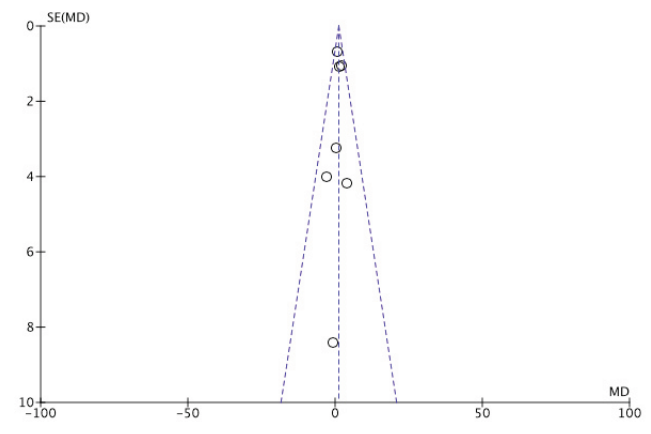

Figure 3A. Funnel plot of overall survival (OS).

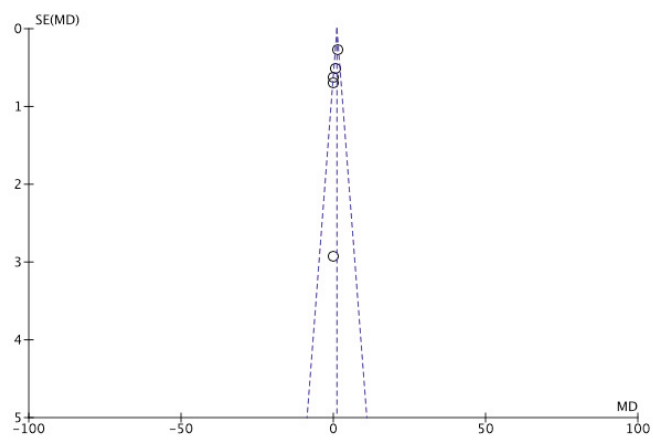

Figure 3B. Funnel plot of progression-free survival (PFS). 


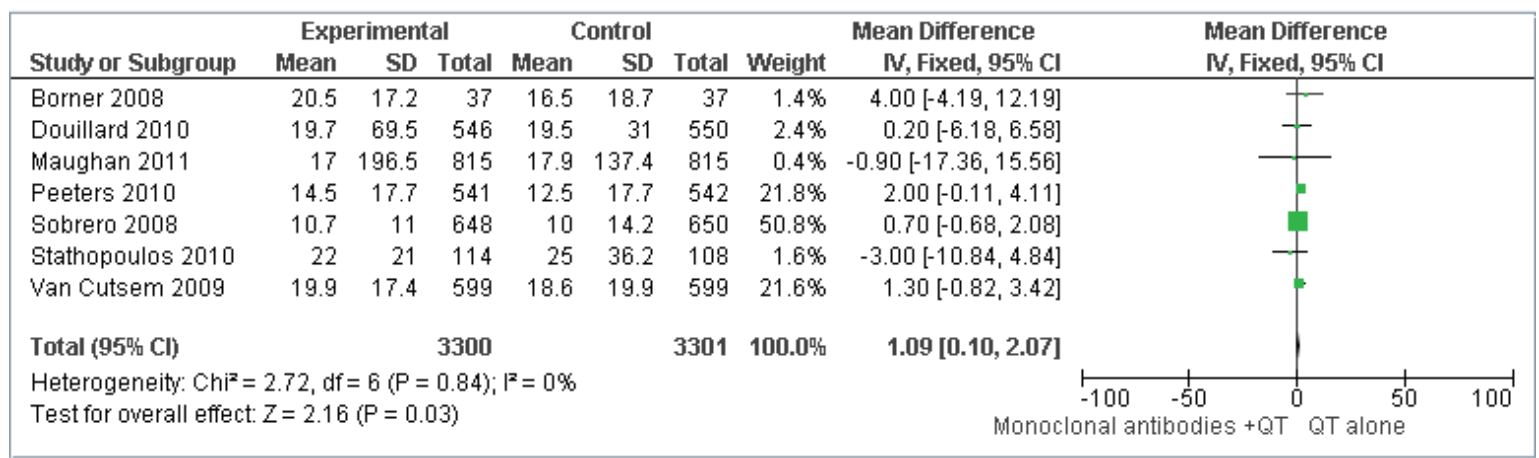

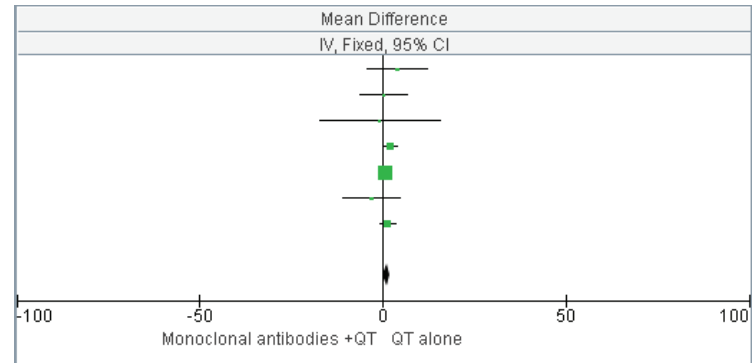

Figure 4. Overall survival (OS)-comparison: MAs + CT versus treatment without MAs.

\begin{tabular}{|c|c|c|c|c|c|c|c|c|c|c|}
\hline \multirow[b]{2}{*}{ Stucty or Subgroup } & \multicolumn{3}{|c|}{ Experimental } & \multicolumn{3}{|c|}{ Control } & \multirow[b]{2}{*}{ Weight } & \multirow{2}{*}{$\begin{array}{l}\text { Mean Difference } \\
\text { N, Random, 95听 Cl }\end{array}$} & \multirow{2}{*}{ 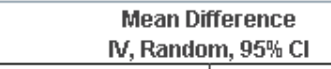 } & \\
\hline & Mean & SD & Total & Mean & SD & Total & & & & \\
\hline Bokemeyer 2009 & 7.2 & 6.9 & 169 & 7.2 & 5.9 & 168 & $16.6 \%$ & $0.00[-1.37,1.37]$ & 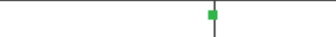 & \\
\hline Douillard 2010 & 8.4 & 10.6 & 546 & 8.4 & 10.1 & 550 & $19.2 \%$ & $0.00[-1.23,1.23]$ & & \\
\hline Maughan 2011 & 8.6 & 63.2 & 815 & 8.6 & 54.5 & 815 & $1.4 \%$ & $0.00[-5.73,5.73]$ & & \\
\hline Sobrero 2008 & 4 & 5.8 & 648 & 2.6 & 3.8 & 650 & $39.0 \%$ & $1.40[0.87,1.93]$ & 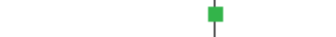 & \\
\hline Van Cutsem 2009 & 8.9 & 9.3 & 599 & 8 & 8.7 & 599 & $23.8 \%$ & $0.90[-0.12,1.92]$ & 1 & \\
\hline Total (95\% Cl) & & & 2777 & & & 2782 & $100.0 \%$ & $0.76[0.08,1.44]$ & & \\
\hline \multicolumn{8}{|c|}{$\begin{array}{l}\text { Heterogeneity: } \operatorname{Tau}^{2}=0.23 ; \mathrm{Chi}^{2}=6.91, \mathrm{df}=4(\mathrm{P}=0.14) ; \mathrm{I}^{2}=42 \% \\
\text { Test for overall effect: } Z=2.20(P=0.03)\end{array}$} & Monocl & $\begin{array}{lcc}-100 \quad-50 & 0 & 50 \\
\text { lonal antibodies+QT } Q T \text { alone }\end{array}$ & 100 \\
\hline
\end{tabular}

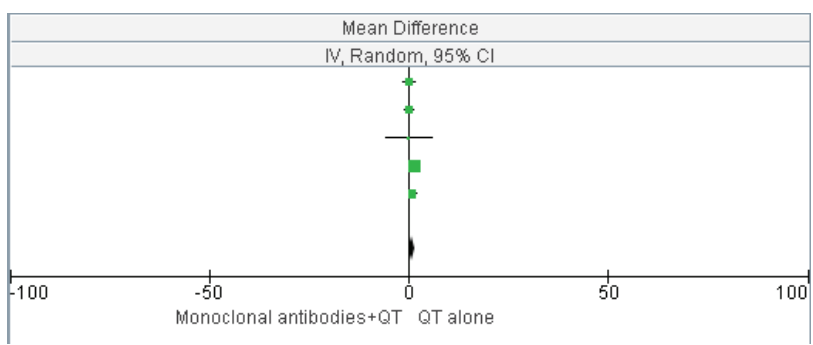

Figure 5. Progression-free survival(PFS)-comparison: MAs + CTversus treatment without MAs. 


\section{Resection rate}

Metastatic resection only was evaluated in two studies [52, 54], both for liver metastases only. The first study reported a statistically significant difference among the 23 radical resections (R0-18 versus 5 - CT + cetuximab versus CT only, respectively-Odds ratio-4.37, $\mathrm{P}<0.01$ ) [54]. Conversely, the second study found no statistically significant difference on hepatic resection rate between treatment groups (4.4\% in the control group (CT + cediranib) versus 5.2\% in the CT + bevacizumab group—Odds ratio, 0,89; 95\% Cl- 0.54-1.46, P = 0.637) [52]. Because of the low percentage of resection cases, the logistic regression model was adjusted by treatment and by the criterion of single liver disease at baseline [52].

\section{Time to progression}

Time to progression was assessed by a single study [49]. In that work, the time to disease progression was 7.2 months (95\% Cl: $6.0-8.4)$ for the intervention group (XELOX + cetuximab), versus 5.8 months (95\% Cl: 5.0-8.3) for the control group (XELOX only). In that case, although the author published the $\mathrm{Cl}$ of the means, a meta-analysis was not performed, as it was the only study that assessed this outcome.

\section{Conversion rate and $\mathrm{QOL}$}

Conversion rate was not assessed by any of the RCTs. QOL was assessed in only two studies [39, 48].

\section{KRAS}

\section{Subgroup analysis of KRAS oncogene status}

Five studies compared tumour response rate in subgroups stratified by KRAS status [42-45, 47]. However, one was excluded because although the author reported that PFS, response rate, and toxicity had been assessed in each group of KRAS status, the results of the mutated KRAS group were not published, which prevented any comparison and characterised selective reporting [44]. In the other studies, the groups with wild-type KRAS that received MA + CT had better results in every RCTs; in two of them, the difference was statistically significant $[42,45]$. Conversely, for participants with mutated KRAS, the addition of MA to CT did not lead to better results when compared with CT alone (Table 1). Only one study (OPUS) conducted this type of evaluation regarding radical metastatic resection (R0) [47]. Similarly, patients with wild-type KRAS who received MA + CT had better results than those who have received CT only with twice the number of resections [47]. The author did not publish the statistical significance for this difference.

Again, the $\mathrm{Cl}$ of the means were not published in those RCTs, which prevented the inclusion of these studies in the meta-analysis.

\section{Discussion}

OS is a universally accepted hard outcome to assess cancer treatment results, and is the main outcome in the process of validation and approval of new drugs. PFS is not statistically validated as a surrogate outcome for survival in all scenarios; however, in the present study, there was no difference in response between the two groups when comparing the two types of outcome. For the OS outcome, the addition

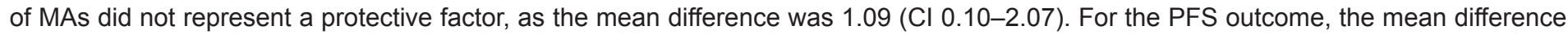
was 0.76 ( $\mathrm{Cl} 0.08-1.44)$, which indicates a health protection factor, although a statistically significant difference was not observed. An argument that could compromise these results would be the presence of heterogeneity between primary studies. However, this was considered to be at the best moderate. Although the funnel plots did not present dispersion, a range of $0-42 \%$ was observed when using the $I^{2}$ statistic indicating respectively, low and moderate levels of inconsistency for OS and PFS. 
When the analysis was focused on the type of KRAS, comparing the results of tumour response rate in participants who had the wild-type gene versus the mutated gene, the available results favoured wild-type KRAS (Table 1). KRAS is an oncogene, located on chromosome 6 , which encodes a protein with an important physiological and pathogenic role in metastatic colorectal cancer (a key protein in EGFR signalling cascade). When certain mutations that may be present in tumours occur, especially in codons 12 and 13 of the KRAS gene, protein activation takes place regardless of the presence of EGFR, which is the target of some drugs for the treatment of this type of cancer, such as panitumumab and cetuximab. That is mutated KRAS has the potential to prevent the mechanism of action of the main drugs that treat metastatic colorectal cancer, which may explain the low results and the absence of a statistically significant difference observed in the mutated KRAS subgroup (Table 1).

Although this meta-analysis was based on high-quality methodologically correct RCTs, the present study had limitations. Its findings and interpretations were limited by the quality and quantity of information available. The non-inclusion of more recent and/or unpublished studies is a potential selection bias. Publication bias was the main problem observed, especially for conducting the analyses. The allocation concealment method was not determined in any of 16 RCTs included. Some authors did not publish the $\mathrm{Cl}$ of the means of the outcomes of OS, PFS, and time to progression, which made the estimation of standard deviations (SD) and the inclusion of the RCTs in the metaanalysis impossible [39-42, 50,52]. As they were drug studies, the nature of the evaluated interventions allows for blinding which would reduce the likelihood of performance bias.

Trials with various experimental groups can pose a potential problem. Many studies are designed to compare more than two treatments. In these cases, there may be two or more experimental interventions, for instance, a drug in different doses, or variations in the therapeutic schemes associated with that intervention. It may be inappropriate to combine heterogeneous intervention groups and compare them with the control group, since it will impair the confidence regarding that result. When there is only one control group, it could be divided; however, to combine these results in the same meta-analysis is inappropriate, because they will be counted twice.

According to The Grades of Recommendation, Assessment, Development and Evaluation Working Group (GRADE) [120] an evidence quality rating system, the estimate of the effect decreases if the studies present severe limitations in their design and implementation that can result in a partial assessment of the effects of the intervention. For RCTs, these methodological limitations include important issues such as lack of confidentiality in the allocation, lack of blinding (especially in studies with subjective outcomes that are highly susceptible to biased evaluation), large losses to follow-up, early study termination, or even selective reporting of results among others. Grade is an important tool that defines the quality of a body of evidence as a measure that ensures that an effects or association estimation is close to the specific quantity of interest.

In the early 1980s, the FDA determined that drugs approved for the treatment of cancer should be based on more direct evidence of clinical benefit, such as improvement in survival, in the QOL, or in the symptoms related to the tumour. This study aimed to verify such evidence for MAs in the treatment of metastatic colorectal cancer. Although this study included only patients with metastatic disease, Maughan's RCT (2011) [44] was included in the analysis, as only $2 \%$ of that sample (13 patients) did not have metastasis at any site. The selected studies did not allow for conversion analysis, in total nor in the KRAS status subgroup.

Table 1. Subgroup analysis by KRAS status regarding tumour response rate.

\begin{tabular}{|c|c|c|c|c|c|c|c|c|}
\hline \multirow[t]{3}{*}{ Study } & \multicolumn{8}{|c|}{ Response rate (\%) } \\
\hline & \multicolumn{2}{|c|}{ Wild-type KRAS } & \multirow[t]{2}{*}{ Odds ratio } & \multirow[t]{2}{*}{$\mathbf{P}$} & \multicolumn{2}{|c|}{ Mutated KRAS } & \multirow[t]{2}{*}{ Odds ratio } & \multirow[t]{2}{*}{$\mathbf{P}$} \\
\hline & $M A+C T$ & CT & & & $M A+C T$ & CT & & \\
\hline Peeters ${ }^{38}$ & 35 & 10 & - & 0.001 & 13 & 14 & - & - \\
\hline Douillard $^{39}$ & 55 & 48 & 1.35 & 0.068 & 40 & 40 & - & - \\
\hline Van Cutsem $^{41}$ & 59.3 & 43.2 & 1.91 & 0.03 & 36.2 & 40.2 & 0.80 & - \\
\hline Bokemeyer $^{43}$ & 3 & 1 & 2.54 & 0.11 & 0 & 2 & 0.50 & 0.106 \\
\hline
\end{tabular}




\section{Conclusion}

In conclusion, this meta-analysis demonstrated that the addition of MAs (bevacizumab, panitumumabe, or cetuximab) to OX or irinotecanbased chemotherapy schemes in first-line treatment in patients with resectable liver metastasis did not increase OS or PFS. Because of the heterogeneity of the studies, particularly regarding KRAS status, prospective controlled clinical trials to study the combination of drugs are needed.

\section{Conflicts of interest}

The authors declare no conflicts of interest.

\section{References}

1. Cancer Facts and Figures (2011) American Cancer Society Atlanta

2. Van Roosbroeck S, Hoeck S and Van Hal G (2012) Population-based screening for colorectal cancer using an immunochemical fecal occult blood test: A comparison of two invitation strategies Cancer Epidemiol 36(5) 317-24 DOI: 10.1016/j. canep.2012.04.003

3. Elias E et al (2012) Lymph node ratio is an independent prognostic factor in Stage III colorectal cancer patients: a retrospective study from the Middle East World J Surg Oncol 10 63-70 DOI: 10.1186/1477-7819-10-63

4. Deschoolmeester $\mathrm{V}$ et al (2010) A review of the most promising biomarkers in colorectal cancer: one step closer to targeted therapy Oncologist 15(7) 699-731 DOI: 10.1634/theoncologist.2010-0025 PMID: 20584808 PMCID: $\underline{3228001}$

5. Figueras $\mathrm{J}$ et al (2013) Preoperative treatment with bevacizumab in combination with chemotherapy in patients with unresectable metastatic colorectal carcinoma Clin Trans/ Oncol 15(6) 460-6 DOI: 10.1007/s12094-012-0952-6

6. Papa A et al (2012) Emerging role of cetuximab in the treatment of colorectal cancer Recent Pat Anticancer Drug Discov 7(2) 233-47 DOI: 10.2174/157489212799972882 PMID: 22264223

7. Satram-Hoang S et al (2013) Comparative Effectiveness of Chemotherapy in Elderly Patients with Metastatic Colorectal Cancer J Gastrointest Cancer 44(1) 79-88 DOI: 10.1007/s12029-012-9450-x PMCID: 3568483

8. Incidência de câncer no Brazil (2013) Instituto Nacional de Câncer José Alencar Gomes da Silva, Coordenação Geral de Ações Estratégicas Coordenação de Prevenção e Vigilância-Rio de Janeiro Brazil

9. Michor F et al (2005) Dynamics of colorectal cancer Semin Cancer Biol 15(6) 484-93 DOI: 10.1016/j.semcancer.2005.06.005 PMID: 16055342

10. Tol J and Punt CJ (2010) Monoclonal antibodies in the treatment of metastatic colorectal cancer: a review Clinical Therapeutics 32(3) 437-53 DOI: 10.1016/j. clinthera.2010.03.012 PMID: 20399983

11. Wang L, Chen X, Li W and Sheng Z (2012) Antiepidermal growth factor receptor monoclonal antibody improves survival outcomes in the treatment of patients with metastatic colorectal cancer Anti-Cancer Drugs 23(2) 155-60 DOI: 10.1097/ CAD.0b013e32834c3256

12. Benoist $S$ and Nordlinger B (2009) The role of preoperative chemotherapy in patients with resectable colorectal liver metastases Ann Surg Oncol 2009 16(9) 2385-90 DOI: 10.1245/s10434-009-0492-7 PMID: 19554377 
13. Herbertson RA et al (2012) Epidermal growth factor receptor (EGF-R) inhibitors for metastatic colorectal cancer (Protocol for a Cochrane Review) The Cochrane Library Issue 11

14. Peeters M et al (2012) Panitumumab in Combination With Cytotoxic Chemotherapy for the Treatment of Metastatic Colorectal Carcinoma Clinical Colorectal Cancer 11(1) 14-23 DOI: 10.1016/j.clcc.2011.06.010

15. Wagner Anna DADW et al (2012) Anti-angiogenic therapies for metastatic colorectal cancer. Cochrane Database of Systematic Reviews in The Cochrane Library Issue 12

16. Douillard JY, Bennouna J and Senellart H (2008) Is XELOX equivalent to FOLFOX or other continuous-infusion 5-fluorouracil chemotherapy in metastatic colorectal cancer? Clin Color Cancer 7(3) 206-11 DOI: 10.3816/CCC.2008.n.029

17. Nordlinger B et al (2008) Perioperative chemotherapy with FOLFOX4 and surgery versus surgery alone for resectable liver metastases from colorectal cancer (EORTC Intergroup trial 40983): a randomised controlled trial Lancet 371(9617) 1007-16 DOI: 10.1016/S0140-6736(08)60455-9 PMID: 18358928 PMCID: 2277487

18. Boostrom SY et al (2009) Impact of neoadjuvant chemotherapy with FOLFOX/FOLFIRI on disease-free and overall survival of patients with colorectal metastases J Gastrointest Surg 13(11) 2003-9 DOI: 10.1007/s11605-009-1007-3 PMID: 19760306 PMCID: $\underline{2813967}$

19. Chibaudel B et al (2012) Therapeutic strategy in unresectable metastatic colorectal cancer Ther Adv Med Oncol 4(2) 75-89 DOI: 10.1177/1758834011431592 PMID: 22423266 PMCID: $\underline{3296081}$

20. Huang MY et al (2011) Prospective analysis of KRAS wild-type patients with metastatic colorectal cancer using cetuximab plus FOLFIRI or FOLFOX4 treatment regimens Genet Mol Res 10(4) 3002-12 DOI: 10.4238/2011.October.3.4 PMID: $\underline{21968808}$

21. Stintzing $S$ et al (2009) The treatment of colorectal carcinoma with monoclonal antibodies. The importance of KRAS mutation analysis and EGFR status Dtsch Arztebl Int 106(12) 202-6 PMID: 19471640 PMCID: 2680580

22. Broadbridge VT, Karapetis CS and Price TJ (2012) Cetuximab in metastatic colorectal cancer Expert Rev Anticancer Ther 12(5) 555-65 DOI: 10.1586/era.12.25 PMID: 22594891

23. Cunningham $D$ et al (2004) Cetuximab monotherapy and cetuximab plus irinotecan in irinotecan-refractory metastatic colorectal cancer N Engl J Med 351(4) 337-45 DOI: 10.1056/NEJMoa033025 PMID: 15269313

24. García-Foncillas J and Díaz-Rubio E (2010) Progress in metastatic colorectal cancer: growing role of cetuximab to optimize clinical outcome Clin Transl Oncol 12(8) 533-42 DOI: 10.1007/s12094-010-0551-3 PMID: 20709651

25. Brink M et al (2003) K-ras oncogene mutations in sporadic colorectal cancer in The Netherlands Cohort Study Carcinogenesis 24(4) 703-10 DOI: $10.1093 / \mathrm{carcin/bgg009}$ PMID: 12727799

26. Kressner $U$ et al (1998) Ki-ras mutations and prognosis in colorectal cancer Eur J Cancer 34(4) 518-21 DOI: 10.1016/S09598049(97)10111-3 PMID: $\underline{9713302}$

27. Bos JL (1990) Ras oncogenes in human cancer: a review Cancer Res 49(17) 4682-9

28. Fearon ER and Vogelstein B (1990) A genetic model for colorectal tumorigenesis Cell 61(5) 759-67 DOI: 10.1016/00928674(90)90186-I PMID: 2188735

29. Jonker DJ et al (2007) Cetuximab for the treatment of colorectal cancer. N Engl J Med 357(20) 2040-8 DOI: 10.1056/NEJMoa071834 PMID: 18003960

30. Keating GM (2010) Panitumumab: a review of its use in metastatic colorectal cancer Drugs 70(8) 1059-78 DOI: 10.2165/11205090000000000-00000 PMID: $\underline{20481659}$ 
31. Giusti RM etal (2008) US Food and Drug Administration approval: panitumumab for epidermal growth factor receptor expressing metastatic colorectal carcinoma with progression following fluoropyrimidine, oxaliplatin, and irinotecan-containing chemotherapy regimens Clin Cancer Res 14(5) 1296-302 DOI: 10.1158/1078-0432.CCR-07-1354 PMID: 18316547

32. Tappenden $\mathrm{P}$ et al (2007) Systematic review and economic evaluation of bevacizumab and cetuximab for the treatment of metastatic colorectal cancer Health Technol Assess 11(12) 1-128 DOI: 10.3310/hta11120 PMID: 17346499

33. Bennouna $\mathrm{J}$ et al (2013) Continuation of bevacizumab after first progression in metastatic colorectal cancer (ML18147): a randomized phase 3 trial Lancet Oncol 14(1) 29-37 DOI: 10.1016/S1470-2045(12)70477-1

34. Masi G et al (2010) Bevacizumab with FOLFOXIRI (irinotecan, oxaliplatin, fluorouracil, and folinate) as first-line treatment for metastatic colorectal cancer: a phase 2 trial Lancet Oncol 11(6) 845-52 DOI: 10.1016/S1470-2045(10)70175-3 PMID: 20702138

35. Bidard FC et al (2010) Single circulating tumor cell detection and overall survival in nonmetastatic breast cancer Ann Oncol 21(4) 729-33 DOI: 10.1093/annonc/mdp391

36. Saad ED and Katz A (2009) Progression-free survival and time to progression as primary end points in advanced breast cancer: often used, sometimes loosely defined Ann Oncol 20(3) 460-4 DOI: 10.1093/annonc/mdn670

37. Eisenhauer EA et al (2009) New response evaluation criteria in solid tumours: revised RECIST guideline (version1.1) Eur $J$ Cancer 45(2) 228-47 DOI: 10.1016/j.ejca.2008.10.026

38. metaRegister of Controlled Trials (mRCT) [www.controlled-trials.com/mrct/] date accessed: 3 March 2014

39. Hurwitz $\mathrm{H}$ et al (2004) Bevacizumab plus irinotecan, fluorouracil, and leucovorin for metastatic colorectal cancer N Engl J Med 350(23) 2335-42 DOI: 10.1056/NEJMoa032691 PMID: 15175435

40. Kabbinavar $\mathrm{F}$ et al (2005) Addition of bevacizumab to bolus fluorouracil and leucovorin in first-line metastatic colorectal cancer: results of a randomized Phase II trial J Clin Oncol 23(16) 3697-705 DOI: 10.1200/JCO.2005.05.112 PMID: 15738537

41. Saltz LB et al (2008) Bevacizumab in combination with oxaliplatin-based chemotherapy as first-line therapy in metastatic colorectal cancer: a randomized phase III study J Clin Oncol 26(12) 2013-9 DOI: 10.1200/JCO.2007.14.9930 PMID: 18421054

42. Peeters $\mathrm{M}$ et al (2010) Randomized phase III study of panitumumab with fluorouracil, leucovorin, and irinotecan (FOLFIRI) compared with FOLFIRI alone as second-line treatment in patients with metastatic colorectal cancer $J$ Clin Oncol 28(31) 4706-13 DOI: 10.1200/JCO.2009.27.6055 PMID: 20921462

43. Douillard JY et al (2010) Randomized, phase III trial of panitumumab with infusional fluorouracil, leucovorin, and oxaliplatin (FOLFOX4) versus FOLFOX4 alone as first-line treatment in patients with previously untreated metastatic colorectal cancer: the PRIME study J Clin Oncol 28(31) 4697-705 DOI: 10.1200/JCO.2009.27.4860 PMID: 20921465

44. Maughan TS et al (2011) Addition of cetuximab to oxaliplatin-based first-line combination chemotherapy for treatment of advanced colorectal cancer: results of the randomised phase 3 MRC COIN trial Lancet 377(9783) 2103-14 DOI: 10.1016/ S0140-6736(11)60613-2 PMID: 21641636 PMCID: $\underline{3159415}$

45. Van Cutsem E et al (2009) Cetuximab and chemotherapy as initial treatment for metastatic colorectal cancer $N$ Engl $J$ Med 360(14) 1408-17 DOI: 10.1056/NEJMoa0805019 PMID: 19339720

46. Adams RA et al (2009) Toxicity associated with combination oxaliplatin plus fluoropyrimidine with or without cetuximab in the MRC COIN trial experience Br J Cancer 100(2) 251-8 DOI: 10.1038/sj.bjc.6604877 PMID: 19165196 PMCID: 2634710

47. Bokemeyer $\mathrm{C}$ et al (2009) Fluorouracil, leucovorin, and oxaliplatin with and without cetuximab in the first-line treatment of metastatic colorectal cancer J Clin Oncol 27(5) 663-71 DOI: 10.1200/JCO.2008.20.8397

48. Sobrero AF et al (2008) EPIC: phase III trial of cetuximab plus irinotecan after fluoropyrimidine and oxaliplatin failure in patients with metastatic colorectal cancer J Clin Oncol 26(14) 2311-9 DOI: 10.1200/JCO.2007.13.1193 PMID: 18390971 
49. Borner $\mathrm{M}$ et al (2008) Adding cetuximab to capecitabine plus oxaliplatin (XELOX) in first-line treatment of metastatic colorectal cancer: a randomized phase II trial of the Swiss Group for Clinical Cancer Research SAKK Ann Oncol 19(7) 1288-92 DOI: 10.1093/annonc/mdn058 PMID: 18349029

50. Cui $\mathrm{F}$ et al (2009) Clinical research of bevacizumab in combination with irinotecan, fluorouracil and leucovorin for advanced metastatic colorectal cancer Zhonghua Wei Chang Wai Ke Za Zhi 12 374-7 PMID: 19598023

51. Stathopoulos GP et al (2010) Treatment of colorectal cancer with and without bevacizumab: a phase III study Oncology 78(5-6) 376-81 DOI: 10.1159/000320520 PMID: 20798560

52. Schmoll HJ et al (2012) Cediranib with mFOLFOX6 versus bevacizumab with mFOLFOX6 as first-line treatment for patients with advanced colorectal cancer: a double-blind, randomized phase III study (HORIZON III) J Clin Oncol 30(29) 3588-95 DOI: 10.1200/JCO.2012.42.5355 PMID: 22965961

53. Badarinath S et al (2004) Cetuximab plus FOLFOX for colorectal cancer (EXPLORE): Preliminary safety analysis of a randomized phase III trial J Clin Oncol 22 3531-9

54. Ye LC et al (2013) Randomized Controlled Trial of Cetuximab Plus Chemother- apy for Patients With KRAS Wild-Type Unresectable Colorectal Liver-Limited Metastases J Clin Oncol 31(16) 1931-8 DOI: 10.1200/JCO.2012.44.8308 PMID: 23569301

55. Colucci G et al (2010) Cetuximab plus FOLFOX-4 in untreated patients with advanced colorectal cancer: a Gruppo Oncologico dell'Italia Meridionale Multicenter phase II study Oncology 79(5-6) 415-22 DOI: 10.1159/000323279

56. Wong NS et al (2011) A phase II study of capecitabine, oxaliplatin, bevacizumab and cetuximab in the treatment of metastatic colorectal cancer Anticancer Res 31(1) 255-61 PMID: 21273607 PMCID: 4106457

57. Spigel DR et al (2010) Phase II trial of FOLFOX6, bevacizumab, and cetuximab in the first-line treatment of metastatic colorectal cancer Clin Adv Hematol Oncol 8(7) 480-5 498 PMID: 20864916

58. Boccia RV et al (2010) A phase II trial of FOLFOX6 and cetuximab in the first-line treatment of patients with metastatic colorectal cancer Clin Colorectal Cancer 9(2) 102-7 DOI: 10.3816/CCC.2010.n.014 PMID: 20378504

59. Raoul JL et al (2009) Cetuximab in combination with irinotecan/5-fluorouracil/folinic acid (FOLFIRI) in the initial treatment of metastatic colorectal cancer: a multicentre two-part phase I/II study BMC Cancer 9112 DOI: 10.1186/1471-2407-9-112 PMID: 19366444 PMCID: 2678147

60. Tabernero $\mathrm{J}$ et al (2007) Phase II trial of cetuximab in combination with fluorouracil, leucovorin, and oxaliplatin in the first-line treatment of metastatic colorectal cancer J Clin Oncol 25(33) 5225-32 DOI: 10.1200/JCO.2007.13.2183 PMID: 18024868

61. Masi G et al (2010) Bevacizumab with FOLFOXIRI (irinotecan, oxaliplatin, fluorouracil, and folinate) as first-line treatment for metastatic colorectal cancer: a phase 2 trial Lancet Oncol 11(6) 845-52 DOI: 10.1016/S1470-2045(10)70175-3 PMID: 20702138

62. Lenz HJ et al (2004) Activity of cetuximab in patients with colorectal cancer refractory to both irinotecan and oxaliplatin $\mathrm{J}$ Clin Oncol 22 3510-14

63. Assenat $\mathrm{E}$ et al (2011) Cetuximab plus FOLFIRINOX (ERBIRINOX) as first-line treatment for unresectable metastatic colorectal cancer: a phase II trial Oncologist 16(11)1557-64 DOI: 10.1634/theoncologist.2011-0141 PMID: 22016477 PMCID: 3233290

64. Uehara $\mathrm{K}$ et al (2011) Conversion chemotherapy using cetuximab plus FOLFIRI followed by bevacizumab plus mFOLFOX6 in patients with unresectable liver metastases from colorectal cancer Jpn J Clin Oncol 41(10) 1229-32 DOI: 10.1093/jijco/hyr115 PMID: 21840871

65. Carneiro BA et al (2012) Phase II study of irinotecan and cetuximab given every 2 weeks as second-line therapy for advanced colorectal cancer Clin Colorectal Cancer 11(1) 53-9 DOI: 10.1016/j.clcc.2011.05.003

66. Kang MJ et al (2012) Biweekly cetuximab plus irinotecan as second-line chemotherapy for patients with irinotecan-refractory and KRAS wild-type metastatic colorectal cancer according to epidermal growth factor receptor expression status Invest New Drugs 30(4) 1607-13 DOI: $10.1007 / \mathrm{s} 10637-011-9703-8$ 
67. Ibrahim EM et al (2007) Safety and efficacy of cetuximab-chemotherapy combination in Saudi patients with metastatic colorectal cancer Indian J Cancer 44(2) 256-61 DOI: 10.4103/0019-509X.35812

68. Sobrero A et al (2009) Phase IV study of bevacizumab in combination with infusional fluorouracil, leucovorin and irinotecan (FOLFIRI) in first-line metastatic colorectal cancer Oncology 77(2) 113-9 DOI: 10.1159/000229787 PMID: 19628950

69. Van Cutsem E et al (2008) An open-label, single-arm study assessing safety and efficacy of panitumumab in patients with metastatic colorectal cancer refractory to standard chemotherapy Ann Oncol 19(1) 92-8 DOI: 10.1093/annonc/mdm399

70. Meyerhardt JA et al (2007) Phase II study of FOLFOX, bevacizumab and erlotinib as first-line therapy for patients with metastatic colorectal cancer Ann Oncol 18(7) 1185-9 DOI: 10.1093/annonc/mdm124 PMID: 17483115

71. Martín-Martorell $P$ et al (2008) Biweekly cetuximab and irinotecan in advanced colorectal cancer patients progressing after at least one previous line of chemotherapy: results of a phase II single institution trial Br J Cancer 99(3) 455-8 DOI: 10.1038/ sj.bjc.6604530 PMID: 18665167 PMCID: 2527794

72. Bennouna J et al (2012) Bevacizumab combined with chemotherapy in the second-line treatment of metastatic colorectal cancer: results from the phase II BEVACOLOR study Clin Colorectal Cancer 11(1) 38-44 DOI: 10.1016/j.clcc.2011.05.002

73. Saltz LB et al (2004) Phase II trial of cetuximab in patients with refractory colorectal cancer that expresses the epidermal growth factor receptor J Clin Oncol 22(7) 1201-8 DOI: 10.1200/JCO.2004.10.182 PMID: 14993230

74. Galal KM, Khaled Z and Mourad AM (2011) Role of cetuximab and sorafenib in treatment of metastatic colorectal cancer Indian J Cancer 48(1) 47-54 DOI: 10.4103/0019-509X.75825 PMID: 21248446

75. Cunningham $D$ et al (2004) Cetuximab monotherapy and cetuximab plus irinotecan in irinotecan-refractory metastatic colorectal cancer N Engl J Med 351(4) 337-45 DOI: 10.1056/NEJMoa033025 PMID: 15269313

76. Van Cutsem E et al (2012) Intrapatient cetuximab dose escalation in metastatic colorectal cancer according to the grade of early skin reactions: the randomized EVEREST study J Clin Oncol 30(23) 2861-8 DOI: 10.1200/JC0.2011.40.9243 PMID: $\underline{22753904}$

77. Moosmann $\mathrm{N}$ et al (2011) Cetuximab plus capecitabine and irinotecan compared with cetuximab plus capecitabine and oxaliplatin as first-line treatment for patients with metastatic colorectal cancer: AIO KRK-0104 - A randomized trial of the German AIO CRC study group J Clin Oncol 29(8) 1050-8 DOI: 10.1200/JC0.2010.31.1936 PMID: 21300933

78. Tol J et al (2009) Chemotherapy, bevacizumab, and cetuximab in metastatic colorectal cancer N Engl J Med 360(6) 563-72 DOI: 10.1056/NEJMoa0808268 PMID: 19196673

79. Tol J et al (2008) A randomised phase III study on capecitabine, oxaliplatin and bevacizumab with or without cetuximab in first-line advanced colorectal cancer, the CAIRO2 study of the Dutch Colorectal Cancer Group (DCCG). An interim analysis of toxicity Ann Oncol 19(4) 734-8 DOI: 10.1093/annonc/mdm607 PMID: 18272912

80. Saltz LB et al (2007) Randomized phase II trial of cetuximab, bevacizumab, and irinotecan compared with cetuximab and bevacizumab alone in irinotecan-refractory colorectal cancer: the BOND-2 study J Clin Oncol 25(29) 4557-61 DOI: 10.1200/ $\underline{\text { JCO.2007.12.0949 PMID: } 17876013}$

81. Hecht JR et al (2009) A randomized phase IIIB trial of chemotherapy, bevacizumab, and panitumumab compared with chemotherapy and bevacizumab alone for metastatic colorectal cancer J Clin Oncol 27(5) 672-80 DOI: 10.1200/JCO.2008.19.8135

82. Lacouture ME et al (2010) Skin toxicity evaluation protocol with panitumumab (STEPP), a phase II, open-label, randomized trial evaluating the impact of a pre-emptive skin treatment regimen on skin toxicities and quality of life in patients with metastatic colorectal cancer J Clin Oncol 28(8) 1351-7 DOI: 10.1200/JCO.2008.21.7828 PMID: 20142600

83. Giessen C et al (2010) A randomized, phase III trial of capecitabine plus bevacizumab (Cape-Bev) versus capecitabine plus irinotecan plus bevacizumab (CAPIRI-Bev) in first-line treatment of metastatic colorectal cancer: the AIO KRK 0110 trial/ ML22011 trial BMC Cancer 11 367-78 DOI: 10.1186/1471-2407-11-367 
84. Tebbutt NC et al (2010) Capecitabine, bevacizumab, and mitomycin in first-line treatment of metastatic colorectal cancer: results of the Australasian Gastrointestinal Trials Group Randomized Phase III MAX Study J Clin Oncol 28(19) 3191-8 DOI: $\underline{10.1200 / J C O .2009 .27 .7723}$ PMID: $\underline{20516443}$

85. Saltz L et al (2012) Phase III trial of cetuximab, bevacizumab, and 5-fluorouracil/leucovorin vs. FOLFOX-bevacizumab in colorectal cancer Clin Colorectal Cancer 11(2) 101-11 DOI: 10.1016/j.clcc.2011.05.006

86. Stintzing $S$ et al (2012) FOLFIRI plus cetuximab versus FOLFIRI plus bevacizumab as first-line treatment for patients with metastatic colorectal cancer-subgroup analysis of patients with KRAS: mutated tumours in the randomised German AIO study KRK-0306 Ann Oncol 23(7) 1693-9 DOI: 10.1093/annonc/mdr571 PMID: 22219013

87. Zhi J et al (2011) A multicenter, randomized, open-label study to assess the steady-state pharmacokinetics of bevacizumab given with either XELOX or FOLFOX-4 in patients with metastatic colorectal cancer Cancer Chemother Pharmacol 68(5) 1199-206 DOI: 10.1007/s00280-011-1606-z PMID: 21409383

88. Azvolinsky A (2011) Oral anti-angiogenesis treatment plus chemotherapy is not more efficacious than bevacizumab plus chemotherapy in metastatic colorectal cancer Oncology 25(12) 1232-5

89. Tsutsumi S et al Phase II trial of chemotherapy plus bevacizumab as second-line therapy for patients with metastatic colorectal cancer that progressed on bevacizumab with chemotherapy: the Gunma Clinical Oncology Group (GCOG) trial 001 SILK study Oncology 83(3) 151-7 PMID: 22889925

90. Díaz-Rubio E et al (2012) First-line XELOX plus bevacizumab followed by XELOX plus bevacizumab or single-agent bevacizumab as maintenance therapy in patients with metastatic colorectal cancer: the phase III MACRO TTD study Oncologist 17(1) 15-25 DOI: $10.1634 /$ theoncologist.2011-0249

91. Wolff RA et al (2012) A double-blind, randomized, placebo-controlled, phase 2 study of maintenance enzastaurin with 5-fluorouracil/leucovorin plus bevacizumab after first-line therapy for metastatic colorectal cancer Cancer 118(17) 4132-8 DOI: 10.1002/cncr.26692 PMID: 22213153

92. Kato $\mathrm{S}$ et al (2012) Safety verification trials of mFOLFIRI and sequential IRIS + bevacizumab as first- or second-line therapies for metastatic colorectal cancer in Japanese patients Oncology 83(2) 101-7 DOI: 10.1159/000339541 PMID: 22777333

93. Mitchell EP et al (2011) The efficacy and safety of panitumumab administered concomitantly with FOLFIRI or Irinotecan in second-line therapy for metastatic colorectal cancer: the secondary analysis from STEPP (Skin Toxicity Evaluation Protocol With Panitumumab) by KRAS status Clin Colorectal Cancer 10(4) 333-9 DOI: 10.1016/j.clcc.2011.06.004 PMID: 22000810

94. Pectasides D et al (2012) XELIRI-bevacizumab versus FOLFIRI-bevacizumab as first-line treatment in patients with metastatic colorectal cancer: a Hellenic Cooperative Oncology Group phase III trial with collateral biomarker analysis BMC Cancer 12 271-81 DOI: 10.1186/1471-2407-12-271 PMID: 22748098 PMCID: $\underline{3466131}$

95. Giantonio BJ et al (2007) Bevacizumab in combination with oxaliplatin, fluorouracil, and leucovorin (FOLFOX4) for previously treated metastatic colorectal cancer: results from the Eastern Cooperative Oncology Group Study E3200 J Clin Oncol 25(12) 1539-44 DOI: 10.1200/JC0.2006.09.6305 PMID: 17442997

96. Kabbinavar F et al (2003) Phase II, randomized trial comparing bevacizumab plus fluorouracil (FU)/leucovorin (LV) with FU/LV alone in patients with metastatic colorectal cancer J Clin Oncol 21(1) 60-5 DOI: $10.1200 / J C 0.2003 .10 .066$

97. Hurwitz $\mathrm{H}$ et al (2003) Bevacizumab (a monoclonal antibody to vascular endothelial growth factor) prolongs survival in firstline colorectal cancer (CRC): results of a phase III trial of bevacizumab in combination with bolus IFL (irinotecan, 5-fluorouracil, leucovorin) as first-line therapy in subjects with metastatic CRC Proc Am Soc Clin Oncol 22906

98. Folprecht $\mathrm{G}$ et al (2010) Tumour response and secondary resectability of colorectal liver metastases following neoadjuvant chemotherapy with cetuximab: the CELIM randomised phase 2 trial Lancet Oncol 11(1) 38-47 DOI: 10.1016/S1470$\underline{\text { 2045(09)70330-4 }}$ 
99. Hurwitz $\mathrm{HI}$ et al (2005) Bevacizumab in combination with fluorouracil and leucovorin: an active regimen for first-line metastatic colorectal cancer J Clin Oncol 23(15) 3502-8 DOI: 10.1200/JC0.2005.10.017 PMID: 15908660

100. Reidy DL et al (2010) Randomized, phase II study of the insulin-like growth factor-1 receptor inhibitor IMC-A12, with or without cetuximab, in patients with cetuximab or panitumumab refractory metastatic colorectal cancer $J$ Clin Oncol 28 4240-6 DOI: 10.1200/JCO.2010.30.4154 PMID: 20713879 PMCID: $\underline{3296668}$

101. Tveit KM et al (2012) Phase III trial of cetuximab with continuous or intermittent fluorouracil, leucovorin, and oxaliplatin (Nordic FLOX) versus FLOX alone in first-line treatment of metastatic colorectal cancer: the NORDIC-VII study J Clin Oncol 30(15) 1755-62 DOI: $\underline{10.1200 / J C O .2011 .38 .0915}$ PMID: 22473155

102. Venook $\mathrm{A}$ et al (2006) Phase III study of irinotecan/5FU/LV (FOLFIRI) or oxaliplatin/5FU/LV (FOLFOX) \pm cetuximab for patients (pts) with untreated metastatic adenocarcinoma of the colon or rectum (MCRC): CALGB 80203 preliminary results $J$ Clin Oncol 243509

103. Hochster HS et al (2008) Safety and efficacy of oxaliplatin and fluoropyrimidine regimens with or without bevacizumab as first-line treatment of metastatic colorectal cancer: results of the TREE Study J Clin Oncol 26(21) 3523-9 DOI: 10.1200/ $\underline{\text { JCO.2007.15.4138 PMID: } 18640933}$

104.Odom D et al (2011) Health-related quality of life and colorectal cancer-specific symptoms in patients with chemotherapyrefractory metastatic disease treated with panitumumab Int $J$ Colorectal Dis 26(2) 173-81 DOI: 10.1007/s00384-010-1112-5 PMCID: $\underline{3024508}$

105. Ocvirk J et al (2010) Cetuximab plus FOLFOX6 or FOLFIRI in metastatic colorectal cancer: CECOG trial World J Gastroenterol 16(25) 3133-43 DOI: 10.3748/wjg.v16.i25.3133 PMID: 20593498 PMCID: 2896750

106. Van Cutsem E et al (2007) Open-label phase III trial of panitumumab plus best supportive care compared with best supportive care alone in patients with chemotherapy-refractory metastatic colorectal cancer J Clin Oncol 25(13) 1658-64 DOI: 10.1200/

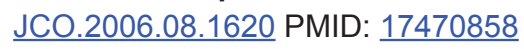

107.Peeters M et al (2009) Association of progression-free survival, overall survival, and patient-reported outcomes by skin toxicity and KRAS status in patients receiving panitumumab monotherapy Cancer 115(7) 1544-54 DOI: 10.1002/cncr.24088 PMID: 19189371

108. Degirmenci $\mathrm{M}$ et al (2010) Efficacy and safety of bevacizumab plus capecitabine and irinotecan regimen for metastatic colorectal cancer Med Oncol 27(3) 585-91 DOI: 10.1007/s12032-009-9253-5

109.Jubb AM et al (2006) Impact of vascular endothelial growth factor-A expression, thrombospondin-2 expression, and microvessel density on the treatment effect of bevacizumab in metastatic colorectal cancer J Clin Oncol 24(2) 217-27 DOI: 10.1200/JCO.2005.01.5388

110. Lin L et al (2010) Evaluation of bevacizumab combined with irinotecan-based regimen as the first-line treatment for patients with metastatic colorectal cancer Zhonghua Zhong Liu Za Zhi 32(10) 786-90 PMID: 21163073

111. Taieb J, Puig PL and Bedenne L (2008) Cetuximab plus FOLFOX-4 for fully resected stage III colon carcinoma: scientific background and the ongoing PETACC-8 trial Expert Rev Anticancer Ther 8(2) 183-9 DOI: 10.1586/14737140.8.2.183 PMID: $\underline{18279058}$

112. Bokemeyer $\mathrm{C}$ et al (2012) Addition of cetuximab to chemotherapy as first-line treatment for KRAS wild-type metastatic colorectal cancer: pooled analysis of the CRYSTAL and OPUS randomised clinical trials Eur J Cancer 48(10) 1466-75 DOI: 10.1016/j.ejca.2012.02.057 PMID: 22446022

113. Ribero $D$ et al (2007) Bevacizumab improves pathologic response and protects against hepatic injury in patients treated with oxaliplatin-based chemotherapy for colorectal liver metastases Cancer 110(12) 2761-7 DOI: 10.1002/cncr.23099 PMID: $\underline{17960603}$ 
114. Bennett $L$ et al (2011) Health-related quality of life in patients with metastatic colorectal cancer treated with panitumumab in first- or second-line treatment Br J Cancer 105(10) 1495-502 DOI: 10.1038/bjc.2011.409 PMID: 21989186 PMCID: $\underline{3242525}$

115. Bokemeyer $\mathrm{C}$ et al (2007) Cetuximab plus 5-FU/FA/oxaliplatin (FOLFOX-4) versus FOLFOX-4 in the first-line treatment of metastatic colorectal cancer (mCRC): OPUS, a randomized phase II study J Clin Oncol 254035

116. Moehler $\mathrm{M}$ et al (2009) Capecitabine and irinotecan with and without bevacizumab for advanced colorectal cancer patients World J Gastroenterol 15(4) 449-56 DOI: 10.3748/wjg.15.449 PMID: 19152449 PMCID: 2653366

117. Alberts SR et al (2012) Effect of oxaliplatin, fluorouracil, and leucovorin with or without cetuximab on survival among patients with resected stage III colon cancer: a randomized trial JAMA 307(13) 1383-93 DOI: 10.1001/jama.2012.385 PMID: 22474202 PMCID: $\underline{3442260}$

118. Madajewicz S et al (2012) Multicenter, randomized phase II trial of bevacizumab plus folinic acid, fluorouracil, gemcitabine (FFG) versus bevacizumab plus folinic acid, fluorouracil, oxaliplatin (FOLFOX4) as first-line therapy for patients with advanced colorectal cancer Invest New Drugs 30(2) 772-8 DOI: 10.1007/s10637-010-9598-9

119. Snoeren N et al (2010) A randomized two arm phase III study in patients post radical resection of liver metastases of colorectal cancer to investigate bevacizumab in combination with capecitabine plus oxaliplatin (CAPOX) vs CAPOX alone as adjuvant treatment BMC Cancer 10545 DOI: 10.1186/1471-2407-10-545 PMID: 20937118 PMCID: 2958953

120. Guyatt GH et al (2011) The GRADE Working Group. GRADE guidelines: 9. Rating up the quality of evidence J Clin Epidemiol 64(12) 1311-6 DOI: 10.1016/j.jclinepi.2011.06.004 PMID: 21802902 